\title{
Expression and clinical significance of interleukin-9 in renal tumors
}

\author{
Zhiyu Zhang", Qi Zhou\#, Jun Ouyang, Jinxian Pu, Jianquan Hou, Jianglei Zhang \\ Department of Urology, the First Affiliated Hospital of Soochow University, Suzhou, China \\ Contributions: (I) Conception and design: J Zhang; (II) Administrative support: J Zhang, J Ouyang; (III) Provision of study materials or patients: \\ Z Zhang, Q Zhou, J Ouyang, J Pu, J Hou; (IV) Collection and assembly of data: Z Zhang, Q Zhou; (V) Data analysis and interpretation: Z Zhang, \\ Q Zhou; (VI) Manuscript writing: All authors; (VII) Final approval of manuscript: All authors. \\ \#These authors contributed equally to this work. \\ Correspondence to: Jianglei Zhang. No. 188, Shizi Street, Gusu District, Suzhou, China. Email: yooseen@126.com.
}

\begin{abstract}
Background: To measure expression levels of interleukin-9 (IL-9) in renal tumors and to determine the clinical significance of those levels.

Methods: Using TCGA database analysis, we found that the expression of IL-9 in renal clear cell carcinoma was significantly down-regulated, and was significantly related to survival. We then verified this using experiments. We enrolled 66 patients who underwent surgical resection of renal tumors between January and December 2018 at the First Affiliated Hospital of Soochow University. Their tumor tissues were paired with adjacent normal tissues and IL-9 expression levels were measured using immunohistochemistry. We determined the correlation of IL-9 expression with clinicopathological features and progression-free survival (PFS).
\end{abstract}

Results: Expression of IL-9 in renal tumors was significantly lower than in adjacent normal tissues $(\mathrm{P}<0.0001)$. There was a significant negative correlation between IL-9 expression levels and R.E.N.A.L. scores $(\mathrm{P}=0.0277)$ as well as with differentiation $(\mathrm{P}=0.0041)$. However, no significant correlation was found between IL-9 levels and clinicopathological features, including gender $(\mathrm{P}=0.0716)$, age $(\mathrm{P}=0.2566)$, body mass index $(\mathrm{BMI})(\mathrm{P}=0.7941)$, tumor size $(\mathrm{P}=0.4193)$ or $\mathrm{TNM}$ staging $(\mathrm{P}=0.5402)$. PFS time in renal tumor patients with positive IL-9 expression was similar to that of patients with negative IL-9 expression.

Conclusions: IL-9 expression was higher in adjacent normal tissues than in renal tumors. Low expression of IL-9 was detected when R.E.N.A.L. score was high or cell differentiation was poor, suggesting that IL-9 may may play a protective role in renal tumor microenvironments.

Keywords: Interleukin-9 (IL-9); renal tumor; immunohistochemistry; prognosis

Submitted Mar 13, 2020. Accepted for publication Sep 26, 2020.

doi: $10.21037 /$ tau-20-761

View this article at: http://dx.doi.org/10.21037/tau-20-761

\section{Introduction}

Renal cell carcinoma (RCC) originates from the epithelial system of renal parenchyma and urinary tubules. Currently, the main treatment for localized RCC is surgical resection. For locally advanced RCC, targeted drug therapy is also necessary. The R.E.N.A.L. score was first proposed by Kutikov et al., to evaluate the difficulty of nephron-sparing surgery (1). It includes five aspects: radius: the diameter of the tumor; exophytic/endophytic rate of the tumor; nearness: the distance between the tumor and the collection system; anterior/posterior: tumor location on the ventral/ visceral side of the kidney; and location: the location of the tumor along the longitudinal axis of the kidney. Higher scores correlate with more difficult nephron-sparing surgery. Nevertheless, there remains $30-40 \%$ of patients who experience metastasis or recurrence after treatment (2). Clear cell renal carcinoma (ccRCC) is the most common 
type of RCC, and some studies have found that destruction of the immune microenvironment is associated with ccRCC occurrence $(3,4)$. For a long time in the past, IL-2 or IFN- $\alpha$ were the main treatments for advanced ccRCC (5-9). Large doses of HDIL-2 can produced toxic effects in some patients (10). This suggests that immunotherapy can be used as a treatment method for ccRCC.

Interleukin-9 (IL-9) is a cytokine secreted mainly by T cells; it has a directional regulatory effect on tumors (11). IL-9 significantly inhibited the growth of melanoma and squamous cell carcinoma in mice $(12,13)$. IL-9 levels were low on pathological examination of colon cancer tissues; however, levels were high in adjacent normal tissues (14), suggesting that IL-9 had an anti-tumor effect. Nevertheless, high expression of IL-9 was measured in cells from liver cancer, pancreatic cancer and chronic lymphoblastic leukemia, suggesting that IL-9 may accelerate the pathogenesis of some tumors $(11,15,16)$. The relationship between IL-9 and ccRCC has not yet been reported. Therefore, the purpose of this study was to measure expression levels of IL-9 in ccRCC and adjacent normal tissue, and to determine correlation between IL-9 expression and clinicopathological characteristics and progression-free survival (PFS). We present the following article/case in accordance with the REMARK reporting checklist (available at http://dx.doi. org/10.21037/tau-20-761).

\section{Methods}

\section{General information}

Database data: the latest transcriptome data and clinical data of 33 kinds of tumors were obtained from the University of California Santa Cruz Xena (https://xena.ucsc.edu/) of The Cancer Genome Atlas database. The Ensembl ID was converted into gene ID, and the gene expression was extracted from the transcriptome data. The expression data were then combined with the survival data of patients with renal clear cell carcinoma. Perl (version 10.0.18362.836) and $\mathrm{R}$ software (version 3.6.2) were used for data processing. Because all data in this study were from TCGA database, patient consent was not required.

Raw data: patients who underwent surgical resection of renal tumors between January and December 2018 at the First Affiliated Hospital of Suzhou University with a pathological diagnosis of ccRCC were enrolled. Inclusion criteria for enrolled patients included complete case information and pathologically confirmed ccRCC after surgery. Exclusion criteria included a history of autoimmune disease or infectious disease. A total of 66 patients were enrolled, including 40 males and 26 females, age [35-82] years, with an average age of $(61.62 \pm 10.87)$. There were 40 patients in which the tumor was less than $4 \mathrm{~cm}$ in diameter, 17 in which the diameters were 4 to $7 \mathrm{~cm}$, and nine patients in which diameters were greater than $7 \mathrm{~cm}$. There were 27 patients with R.E.N.A.L. scores of less than 6 and 37 patients with scores of more than 6 . According to Fuhrman's histological classification, 24, 28, 11, and 3 tumors were classified as grade I, II, III, and IV, respectively. According to TNM staging of American Cancer Federation in 2010, there were 46 tumors in stage I and 20 in stage II. The study was conducted in accordance with the Declaration of Helsinki (as revised in 2013). Our research was approved by the ethics committee of the First Affiliated Hospital of Soochow University (No. 209) and all the patients signed consent forms before the study.

\section{Immunobistochemistry}

\section{Specimen collection}

We collected specimens from renal tumors and corresponding adjacent normal tissues (more than two centimeters away from the edge of the tumor and no invasion of tumor cells confirmed on post-surgical pathology). The specimens were fixed in formalin $(10 \%$ formaldehyde solution) and then embedded in paraffin and sectioned for archiving.

\section{Immunohistochemistry (SP method)}

Preserved paraffin slices were sequentially treated by dewaxing, hydration, phosphate buffer saline (PBS) washing and rinsing with citric acid buffer. To inactivate endogenous peroxidase, $3 \%$ hydrogen peroxide was dropped onto the slices, maintained at room temperature for 10 minutes and washed again in PBS. Subsequently, $5 \%$ bovine serum albumin $50 \mu \mathrm{L}$ were added to the slides and incubated at room temperature for 10 minutes. After adding primary antibody against IL-9 (diluted at 1:200), the slices were incubated overnight at $4{ }^{\circ} \mathrm{C}$, and washed with PBS thereafter. Next, second antibody was added, incubated at room temperature for 60 minutes and then washed with PBS again. Then sections were reacted with DAB color reagent for 5 minutes, re-stained with hematoxylin, dehydrated with a gradient concentration of ethanol, and finally sealed with neutral resin for microscopic observation. 


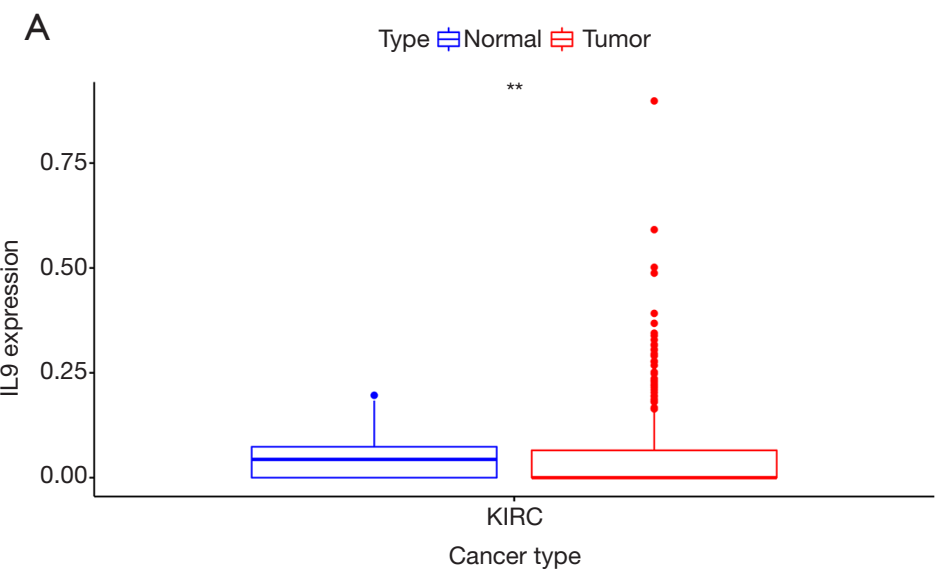

B

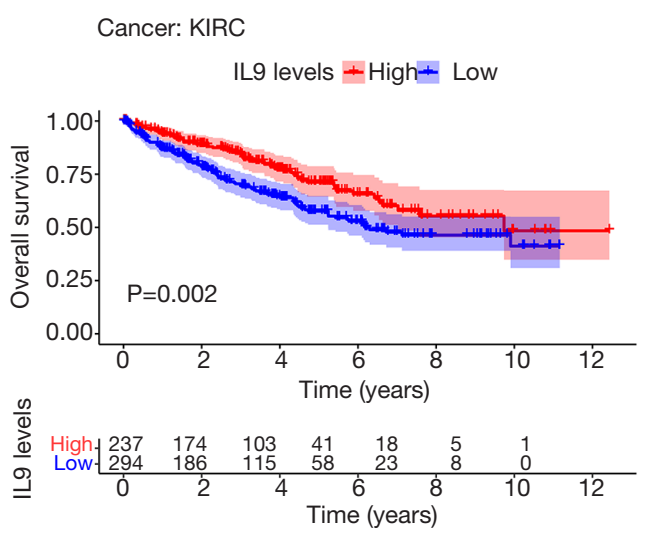

Figure 1 Expression of IL-9 in ccRCC. (A) There was a significant difference in the expression of IL-9 between tumor and adjacent tissues. (B) Effect of IL-9 expression on survival rate of patients. ${ }^{* *}, \mathrm{P}<0.01$. ccRCC, clear cell renal carcinoma; IL-9, interleukin-9.

\section{Criteria for determining results}

Because IL-9 is mainly expressed in the cytoplasm, the appearance of pale yellow, brown yellow or brown granules in the cytoplasm at 200 -fold magnification was considered positive. For a section to be judged positive, ten microscopic views with 400 -fold magnification were randomly observed and scored according to the proportion of positive cells and staining intensity. Specifically, when the proportion of positive staining cells was less than $5 \%, 5-30 \%, 31-60 \%$, and more than $60 \%$, the scores were zero, one, two, and three, respectively. The scoring criteria for staining intensity were as follows: colorless scored zero, light yellow scored one, brown yellow scored two and brown scored three. Finally, the score of positively stained cell proportion was multiplied by the staining intensity score, and the product of less than two points were classified as negative (-); otherwise it was considered positive (+) (14).

\section{Follow-up}

Patients were followed up for six to seven months with the deadline of June 1, 2019. PFS was defined as the time from the day of surgery to the deadline or the time point when progression was observed. Patients with stage T1-T2 were followed up every six weeks after surgery, then every six months for up to three years, and annually thereafter. Patients with stage T3-T4 were followed up every six weeks after surgery, every three months up to two years, then every six months from the third year and annually thereafter. Follow-up was conducted by telephone and outpatient consultation.

\section{Statistical analysis}

Perl software and $\mathrm{R}$ software and related accessory packages. The quantitative data were expressed as mean \pm standard deviation (SD). Qualitative data were expressed as ratios; comparisons between two groups were determined using the Mann-Whitney $U$ test. The comparisons of cumulative data between two groups were examined by $\chi^{2}$ test. Statistical analyses were performed using SPSS 22.0. GraphPad Prism 8.0 was used to draw survival curves. Kaplan-Meier and Log-rank tests were used for survival analysis. All $\mathrm{P}$ values were considered statistically significant if $\mathrm{P}<0.05$.

\section{Results}

\section{Bioinformatics analysis of the expression of interleukin-9} in RCC and survival analysis

It was found that there was a significant difference in the expression of IL-9 between cancer cells and adjacent tissues of patients with renal clear cell carcinoma $(\mathrm{P}<0.05$, Figure $1 A$ ). According to the median value of IL-9, the samples were divided into high and low expression groups. Through difference analysis, we found that there was significant difference in the expression of IL-9 between high and low expression groups. In ccRCC patients, the overall survival rate of the high IL-9 expression group was significantly higher than that of the low expression group, showing a better survival state (Figure 1B, KaplanMeier log-rank test, $\mathrm{P}=0.002$ ). The differential expression graph was generated using the "ggpubr" package, and the 

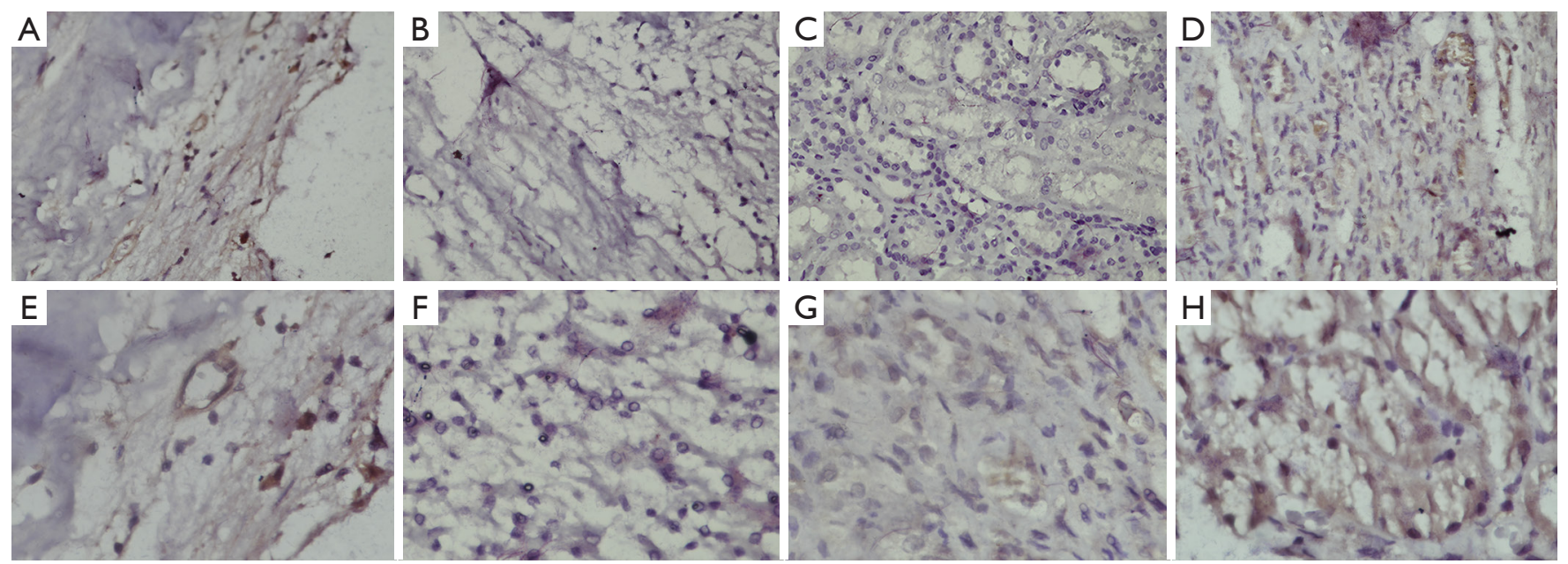

Figure 2 The expression of IL-9 in renal cell carcinoma and adjacent normal tissues. (A) Positive expression of IL-9 in ccRCC (SP*20). (B) Negative expression of IL-9 in ccRCC (SP*20). (C) Positive expression of IL-9 in paracancerous tissues (SP*20). (D) Negative expression of IL-9 in paracancerous tissues (SP*20). (E) Positive expression of IL-9 in ccRCC (SP*40). (F) Negative expression of IL-9 in ccRCC (SP*40). (G) Positive expression of IL-9 in paracancerous tissues (SP*40). (H) Negative expression of IL-9 in paracancerous tissues (SP*40). ccRCC, clear cell renal carcinoma; IL-9, interleukin-9.

Table 1 IL-9 expression in ccRCC and corresponding adjacent normal tissues

\begin{tabular}{lccc}
\hline \multirow{2}{*}{ Tissue } & \multicolumn{2}{c}{ IL-9 expression [case (\%)] } & $\chi^{2}$ \\
\cline { 2 - 3 } & Positive & Negative & P \\
\hline Tumor tissues & $7(10.61)$ & $59(89.39)$ & 88.44 \\
Adjacent tissue & $61(92.42)$ & $5(7.58)$ & $<0.0001$ \\
\hline
\end{tabular}

ccRCC, clear cell renal carcinoma; IL-9, interleukin-9.

survival curve is generated by the "survival" and "survminer" packages.

\section{Expression of IL-9 in ccRCC and adjacent tissues}

Scores of negative and positive expression of IL-9 in cancer tissues were 59 and 7 , respectively, and the positive rate was $10.61 \%$ (Figure 2 and Table 1). By contrast, the scores negative and positive expression of IL-9 in adjacent tissues were 5 and 61, respectively, and the positive rate was $92.42 \%$. This suggests that expression of IL-9 was low in ccRCC tissues but high in adjacent tissues. The difference was statistically significant $(\mathrm{P}<0.0001)$.

\section{Correlation between clinicopathological features and expression of IL-9 in $c \mathrm{RCC}$}

Gender, body mass index (BMI), age, tumor diameter and TNM stage did not correlate with IL-9 expression in renal tumors (Table 2, P>0.05). Patients with R.E.N.A.L. scores $\leq 6$ showed significantly higher IL-9 expression than did patients with R.E.N.A.L. score $>6(\mathrm{P}=0.0277)$. The expression of IL-9 in tumor cells with medium-to-low differentiation was significantly lower than that of those with high differentiation $(\mathrm{P}=0.0041)$.

\section{The correlation between expression of IL-9 and PFS in ccRCC}

Patients were followed up for 5 to 17 months and the follow-up rate was $100 \%$. PFS was $87.88 \%(58 / 66)$ in 66 ccRCC patients until the deadline. PFS of ccRCC patients with negative expression of IL-9 was $88.14 \%$ (52/59), while that of patients with positive expression was $85.71 \%(6 / 7)$. Because of the limited number of patients, univariate survival analysis did not reveal a significant 
Table 2 Correlation between expression of IL-9 and clinicopathological features of ccRCC

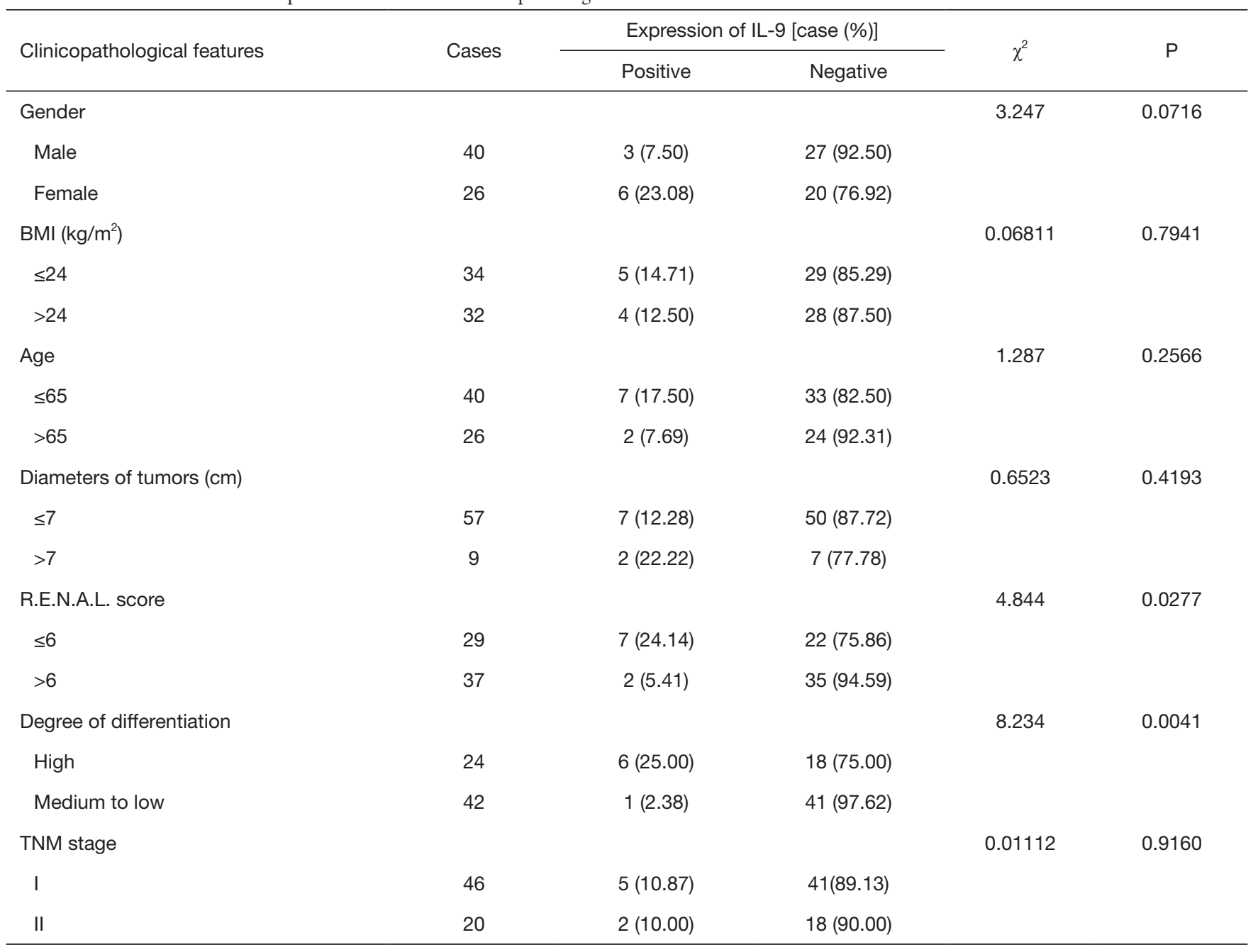

ccRCC, clear cell renal carcinoma; BMI, body mass index; IL-9, interleukin-9.

difference in survival time between patients with positive and negative expression in ccRCC (Kaplan-Meier: $\chi^{2}=0.2301, \mathrm{P}=0.6314$, Log-rank test: $\chi^{2}=0.06847, \mathrm{P}=0.7936$, Figure 3).

\section{Discussion}

Ours is the first study of IL-9 in ccRCC. IL-9 is a cytokine with several biological effects, primarily synthesized by activated Th9 cells and secreted by neutrophils and mast cells in small amounts. IL-9 promotes the transcription and expression of relevant genes in the nucleus and plays a corresponding biological role by activating signal transducers and activator of transcription 1 (STAT1), START3, and STAT5 $(17,18)$. Studies have found that the effector cells of IL-9, including cytotoxic T lymphocytes (CTL), dendritic cells (DC) and mast cells, play important roles in promoting cell inflammation, mediating immune diseases and tumor immunity $(19,20)$.

In this study, we analyzed the data of 537 patients from a database using bioinformatics and found that there were differences in the expression of IL-9 in ccRCC. Furthermore, through survival analysis, we found that the patients with down-regulated expression of IL-9 and downregulated IL-9 had lower survival rates and worse prognosis. In order to verify our inference, a small sample experiment was carried out. Through the experiment, we found that the expression of IL-9 in ccRCC was significantly lower than that in adjacent normal tissues. The relationship between IL-9 and tumors has not been fully clarified. 


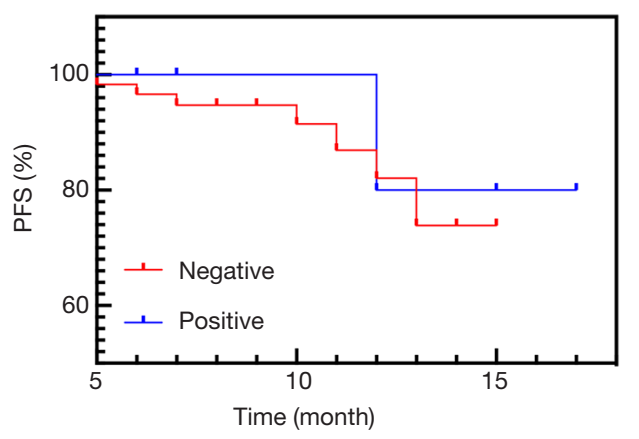

Figure 3 Survival curve of patients with negative and positive IL-9 expression in ccRCC. ccRCC, clear cell renal carcinoma; IL-9, interleukin-9.

On the one hand, IL-9 has been reported to inhibit the growth of melanoma and squamous cell carcinoma (12-14), possibly attributed to IL-9 in stimulating DC cells to enter sentinel lymph nodes, activating CTL cell antigens to initiate and intrude tumor tissues (21). On the other hand, high expression of IL-9 has been measured in the blood of patients with hematological malignancies such as lymphoma (22), and the use of antibodies to block IL-9 with antibodies slowed the progress of tumors (23). These data suggest that IL-9 also promoted tumor progression $(15,16)$. In the present study, IL-9 was expressed at low levels in ccRCC but was highly-expressed in adjacent normal tissues, suggesting that IL-9 may inhibit the growth of ccRCC. This could be explained by the fact that the kidney, an important excretory organ with abundant perfusion, is more conducive to infiltration and aggregation of immune cells, and it secretes a variety of cytokines including IL-9. Nevertheless, ccRCC is mainly composed of renal parenchymal cells, and the immune effector cells in them are in a state of inhibition, resulting in less secretion of IL-9.

In our study, we found that expression of IL-9 in ccRCC was not significantly correlated with clinicopathological features such as gender, age and BMI. However, the influence of gender, age and BMI on the expression of IL-9 still requires further study for confirmation. The TNM stage of renal tumor was associated with tumor diameter in renal tumor. However, there was no significant difference found between the positive expression group and the negative expression group with respect to tumor diameter or TNM stage. This suggests that tumor size may not be associated with the expression of IL-9 in ccRCC. Nevertheless, several related studies found that the expression of IL-9 in peripheral blood of patients with laryngeal squamous cell carcinoma and small cell lung cancer were higher than those of normal controls, and the expression levels of IL-9 in patients with stage III and IV were higher than those of patients with stage I and II, suggesting that IL-9 may correlate with tumor stage $(20,24)$. In the present study, we found that expression levels of IL-9 negatively correlated with the R.E.N.A.L scores and tumor differentiation, suggesting that the R.E.N.A.L. scoring system can be used not only as an auxiliary reference for the evaluation of surgical difficulty of renal tumors, but also for expression of IL-9 in tumors. Considering the limitation of a single center and small sample size in this study, the relationship between the R.E.N.A.L scoring system and IL-9 expression in renal tumors remains to be confirmed in multi-center and large-volume studies. Consistent with previous findings, we found that poor tissue differentiation correlated with lower expression levels of IL-9, suggesting that IL-9 may inhibit occurrence and progression of renal tumors. Future well-designed studies of its potential mechanisms will be also needed to confirm this. Huang et al. found that PFS of colon cancer patients with positive expression of IL-9 was not superior to that of patients with negative expression; nevertheless, the authors found that survival time of patients with high expression of IL-9 was significantly longer than that of patients with low expression (11), suggesting that expression of IL-9 may correlate with survival after surgery. In future studies, we will expand the sample size and extend the follow-up time to further investigate the relationship between IL-9 and PFS.

In summary, IL-9 may have anti-tumor effects on ccRCC; IL-9 levels correlated with R.E.N.A.L. scores and tumor differentiation. The immune mechanism of IL-9 against ccRCC still needs to be investigated. There were some limitations in our study, including the small size and short follow-up period, both of which need to be remedied in the future.

\section{Acknowledgments}

Funding: This work was supported by the Suzhou Science and Technology Project (SLC201906 and SYS2019053).

\section{Footnote}

Reporting Checklist: The authors have completed the REMARK reporting checklist. Available at http://dx.doi. org/10.21037/tau-20-761 
Data Sharing Statement: Available at http://dx.doi. org/10.21037/tau-20-761

Conflicts of Interest: All authors have completed the ICMJE uniform disclosure form (available at http://dx.doi. org/10.21037/tau-20-761). The authors have no conflicts of interest to declare.

Ethical Statement: The authors are accountable for all aspects of the work in ensuring that questions related to the accuracy or integrity of any part of the work are appropriately investigated and resolved. The study was conducted in accordance with the Declaration of Helsinki (as revised in 2013). The study was approved by the ethics committee of the First Affiliated Hospital of Soochow University (No. 209) and informed consent was taken from all individual participants.

Open Access Statement: This is an Open Access article distributed in accordance with the Creative Commons Attribution-NonCommercial-NoDerivs 4.0 International License (CC BY-NC-ND 4.0), which permits the noncommercial replication and distribution of the article with the strict proviso that no changes or edits are made and the original work is properly cited (including links to both the formal publication through the relevant DOI and the license). See: https://creativecommons.org/licenses/by-nc-nd/4.0/.

\section{References}

1. Kutikov A, Uzzo RG. The R.E.N.A.L. nephrometry score: a comprehensive standardized system for quantitating renal tumor size, location and depth. J Urol 2009;182:844-53.

2. Ishibashi K, Koguchi T, Matsuoka K, et al. Interleukin-6 induces drug resistance in renal cell carcinoma. Fukushima J Med Sci 2018;64:103-10.

3. Escudier B, Eisen T, Stadler WM, et al. Sorafenib in advanced clear-cell renal-cell carcinoma. $\mathrm{N}$ Engl J Med 2007;356:125-34.

4. Fan YG, Zhai JM, Wang W, et al. IL-35 over-expression is associated with genesis of gastric cancer. Asian Pac J Cancer Prev 2015;16:2845-9.

5. deKernion JB, Sarna G, Figlin R, et al. The treatment of renal cell carcinoma with human leukocyte alphainterferon. J Urol 1983;130:1063-6.

6. Fisher RI, Rosenberg SA, Fyfe G. Long-term survival update for high-dose recombinant interleukin-2 in patients with renal cell carcinoma. Cancer J Sci Am 2000;6 Suppl 1:S55-7.

7. Fyfe G, Fisher RI, Rosenberg SA, et al. Results of treatment of 255 patients with metastatic renal cell carcinoma who received high-dose recombinant interleukin-2 therapy. J Clin Oncol 1995;13:688-96.

8. Rosenberg SA, Yang JC, White DE, et al. Durability of complete responses in patients with metastatic cancer treated with high-dose interleukin-2: identification of the antigens mediating response. Ann Surg 1998;228:307-19.

9. Yamamoto T, Kitamura H, Masumori N. Complete response to interferon-alpha in a patient with metastatic renal cell carcinoma after unsuccessful molecular-targeted therapies. Int J Urol 2014;21:839-40.

10. McDermott DF, Cheng SC, Signoretti S, et al. The highdose aldesleukin "select" trial: a trial to prospectively validate predictive models of response to treatment in patients with metastatic renal cell carcinoma. Clin Cancer Res 2015;21:561-8.

11. Chen N, Lu K, Li P, et al. Overexpression of IL-9 induced by STAT6 activation promotes the pathogenesis of chronic lymphocytic leukemia. Int J Clin Exp Pathol 2014;7:2319-23.

12. Miao BP, Zhang RS, Sun HJ, et al. Inhibition of squamous cancer growth in a mouse model by Staphylococcal enterotoxin B-triggered Th9 cell expansion. Cell Mol Immunol 2017;14:371-9.

13. Nakatsukasa H, Zhang D, Maruyama T, et al. The DNAbinding inhibitor Id3 regulates IL-9 production in CD4(+) T cells. Nat Immunol 2015;16:1077-84.

14. Huang Y, Cao Y, Zhang S, et al. Association between low expression levels of interleukin-9 and colon cancer progression. Exp Ther Med 2015;10:942-6.

15. Chen N, Lv X, Li P, et al. Role of high expression of IL-9 in prognosis of CLL. Int J Clin Exp Pathol 2014;7:716-21.

16. Tan H, Wang S, Zhao L. A tumour-promoting role of Th9 cells in hepatocellular carcinoma through CCL20 and STAT3 pathways. Clin Exp Pharmacol Physiol 2017;44:213-21.

17. Ma L, Xue HB, Guan XH, et al. Possible pathogenic role of T helper type 9 cells and interleukin (IL)- 9 in atopic dermatitis. Clin Exp Immunol 2014;175:25-31.

18. Zhang L, Wu JH, Otto JC, et al. Interleukin-9 mediates chronic kidney disease-dependent vein graft disease: a role for mast cells. Cardiovasc Res 2017;113:1551-9.

19. Abdul-Wahid A, Cydzik M, Prodeus A, et al. Induction of antigen-specific TH 9 immunity accompanied by mast cell activation blocks tumor cell engraftment. Int J Cancer 
2016;139:841-53.

20. Kim IK, Kim BS, Koh CH, et al. Glucocorticoid-induced tumor necrosis factor receptor-related protein costimulation facilitates tumor regression by inducing IL-9producing helper T cells. Nat Med 2015;21:1010-7.

21. Zhao Y, Chu X, Chen J, et al. Dectin-1-activated dendritic cells trigger potent antitumour immunity through the induction of Th9 cells. Nat Commun 2016;7:12368.

22. Lu Y, Yi Q. Utilizing TH9 cells as a novel therapeutic strategy for malignancies. Oncoimmunology

Cite this article as: Zhang Z, Zhou Q, Ouyang J, Pu J, Hou J, Zhang J. Expression and clinical significance of interleukin-9 in renal tumors. Transl Androl Urol 2020;9(6):2657-2664. doi: 10.21037/tau-20-761 2013;2:e23084.

23. Vieyra-Garcia PA, Wei T, Naym DG, et al. STAT3/5Dependent IL9 Overexpression Contributes to Neoplastic Cell Survival in Mycosis Fungoides. Clin Cancer Res 2016;22:3328-39.

24. Bu XN, Zhou Q, Zhang JC, et al. Recruitment and phenotypic characteristics of interleukin 9-producing CD4+ T cells in malignant pleural effusion. Lung 2013;191:385-9. 